\title{
RESEARCH ANNOUNCEMENTS
}

\section{LEVELS IN ALGEBRA AND TOPOLOGY}

BY Z. D. DAI, T. Y. LAM ${ }^{1}$ AND C. K. PENG

The level $s(A)$ of a (commutative) ring $A$ is the smallest natural number $s$ such that -1 is a sum of $s$ squares in $A$. (If -1 is not a sum of squares in $A$, we say that $s(A)=\infty$.) If $A$ is a field, a striking result of Pfister [3] says that $s(A)$ (if finite) is always a power of 2 , and indeed, all powers of 2 are possible. Knebusch and Baeza have obtained extensions of Pfister's result to semilocal rings, but little is known about levels of commutative rings in general. In [2, Problem 13], Knebusch has asked what type of integers can be the level of a ring (see also $[1$, p. 184]).

In this note, we announce the following.

THEOREM 1A. For any $n \geqslant 1$, there exists an integral domain $A$ with $s(A)=n$. Moreover, $A$ can be chosen so that its field of quotients has any prescribed level $2^{r} \leqslant n$.

A form (homogeneous polynomial) $f \in A\left[x_{1}, \ldots, x_{m}\right]$ is said to be isotropic over $A$ if there exists a unimodular vector $v \in A^{m}$ such that $f(v)=0$. (Otherwise, $f$ is said to be anisotropic over $A$.) Define the sublevel $s^{\prime}(A)$ to be the smallest integer $n$ such that $x_{1}^{2}+\cdots+x_{n+1}^{2}$ is isotropic over $A$. If 2 is invertible in $A$, it is easy to see that $s^{\prime}(A)$ is equal to either $s(A)$ or $s(A)-1$. If $s(A) \in\{1,2,4,8\}$, then in fact $s^{\prime}(A)=s(A)$.

THEOREM 1B. For any $n \geqslant 1$, there exists an integral domain $A$ with $s(A)=s^{\prime}(A)=n$. If $n \geqslant 3$ is odd, there exists an integral domain $B$ with $s(B)$ $=n$ and $s^{\prime}(B)=n-1$.

COROLlARY. The pythagoras number of a ring $A$ (i.e. the smallest integer $r$ such that any sum of squares in $A$ is a sum of $r$ squares) can be any positive integer. (In fact, for the ring $A$ in Theorem $1 \mathrm{~B}$, the polynomial ring $A[t]$ will have pythagoras number $n+1$.)

While the above results are of an algebraic nature, their proofs (at least as so far discovered) are purely topological. One uses ideas from homotopy and

Received by the editors March 28, 1980.

1980 Mathematics Subject Classification. Primary 10J05, 13B25, 55M10; Secondary $10 \mathrm{Cxx}, 10 \mathrm{M05}, 13 \mathrm{~F} 20,55 \mathrm{P} 99$.

${ }^{1}$ Supported in part by NSF. 
cohomotopy theory, and exploits the topology of the real spheres $S^{n}$ and the real Stiefel manifolds $V_{n, 2}$. In the light of their proofs, $1 \mathrm{~A}$ and $1 \mathrm{~B}$ take on the appearance of topological 'dimension theorems'. Indeed, our methods of proof suggest a natural way of carrying over the theory of level and sublevel from algebra and topology, thus exhibiting a new interplay between the two subjects. The topological side of the story seems to be of interest in its own right, and may very well hold the key to other unsolved questions on sums of squares and pythagoras numbers in algebra. To sketch the main ideas involved, we proceed as follows.

Let $\mathcal{C}$ be the category whose objects are topological spaces $X$ equipped with a continuous involution $*$. Morphisms in $C$ are continuous involution-preserving maps. The real spheres $\left(S^{n}, \epsilon\right)$ with the antipodal map $\epsilon$ constitute a distinguished family of objects in $C$. For any $(X, *) \in$ Obj $C$, we define

$$
\begin{gathered}
s(X, *)=\min \left\{n \mid \exists \text { a } C \text {-morphism }(X, *) \rightarrow\left(S^{n-1}, \epsilon\right)\right\} \text { (level), } \\
s^{\prime}(X, *)=\max \left\{n \mid \exists \text { a } C \text {-morphism }\left(S^{n-1}, \epsilon\right) \rightarrow(X, *)\right\} \text { (sublevel). }
\end{gathered}
$$

REMARK. By the Borsuk-Ulam Theorem, we have always $s^{\prime}(X, *) \leqslant$ $s(X, *)$; in particular, $s^{\prime}\left(S^{n-1}, \epsilon\right)=s\left(S^{n-1}, \epsilon\right)=n$.

Another important object in $\mathrm{C}$ is $(\mathbf{C},-)$ with 'bar' = complex conjugation. For any $(X, *)$, let $A_{(X, *)}$ (or $A_{X}$ for short) be the ring of all $\mathrm{C}$-morphisms from $(X, *)$ to $(\mathrm{C},-)$. This ring provides the important link between the algebraic level (resp. sublevel) and the topological level (resp. sublevel), in a way which refines the preceding Remark:

TheOREM 2. For any $(X, *) \in \operatorname{Obj} C$, we have $s^{\prime}(X, *) \leqslant s^{\prime}\left(A_{X}\right) \leqslant s\left(A_{X}\right)$ $=s(X, *)$.

This theorem implies the first part of Theorem 1B for, if we take $(X, *)=$ $\left(S^{n-1}, \epsilon\right)$, then Theorem 2 (plus the Remark) shows that $s^{\prime}\left(A_{X}\right)=s\left(A_{X}\right)=n$.

Another important family of objects in $\mathcal{C}$ is affine varieties $X=V_{\mathbf{C}}(\mathfrak{Q}) \subseteq$ $\mathbf{C}^{n}$ (with the usual topology) defined over the reals by an ideal $\mathscr{U} \subseteq \mathbf{R}\left[x_{1}, \ldots, x_{n}\right]$, with involution 'bar' given by complex conjugation. Let $A=\mathbf{R}\left[x_{1}, \ldots, x_{n}\right] / \mathscr{I}$ be the real coordinate ring of $X$.

THEOREM 3. The following statements are equivalent.

(1) $s(X,-) \leqslant \infty$, (2) $s^{\prime}(X,-)<\infty$, (3) $s\left(A_{X}\right)<\infty$, (4) $s^{\prime}\left(A_{X}\right)<\infty$, (5) $s(A)<\infty$, (6) $s^{\prime}(A)<\infty$, (7) $X$ has no real points. If this is the case, the (sub)levels in (1), (2), (3), (4) are $\leqslant n$.

This follows essentially from the Real Nullstellensatz. To get more precise results, we consider the case of hypersurfaces. 
THEOREM 4. Let $\mathfrak{A}=(g)$ where $g \in \mathbf{R}\left[x_{1}, \ldots, x_{n}\right]$ is absolutely irreducible. Assume that the hypersurface $X=V_{\mathrm{C}}(g)$ has no real points, and that its projective closure is nonsingular. Then

(1) $s^{\prime}(X,-)=s^{\prime}\left(A_{X}\right)=s\left(A_{X}\right)=s(X,-)=n \leqslant s^{\prime}(A) \leqslant s(A)<\infty$.

(2) Let $f \in \mathbf{R}\left[x_{1}, \ldots, x_{n}\right]$ be any real form which is anisotropic over $\mathbf{R}$ Then $f$ remains anisotropic over $A$ and $A_{X}$. In fact, if $f\left(d_{1}, \ldots, d_{n}\right)=0$, $d_{i} \in A$ or $A_{X}$, then the $\left\{d_{j}\right\}$ have a common zero on $X$.

The first part of (2) above has the flavor of a 'Tarski Principle'. The remarkable thing is that such a principle is being affirmed here in a 'nonreal' setting, in which the quotient field of $A$ is not formally real.

The proof of (1) in Theorem 4 relies on the fact that $X$ has the homotopy type of a bouquet of $(n-1)$-spheres. This implies that $X$ is $(n-2)$-connected, from which it is easy to show that $s^{\prime}(X,-) \geqslant n$. The rest of Theorem 4 can then be deduced by an argument involving the topological degree of mappings between spheres.

To convey the general flavor of the methods we used, let us give here a direct proof for the first part of Theorem 1A. We claim that the 'generic' $\mathbf{R}$-algebra $A=\mathbf{R}\left[x_{1}, \ldots, x_{n}\right] /\left(1+x_{1}^{2}+\cdots+x_{n}^{2}\right)$ has level $n$. (This is, of course, a special case of Theorem 4.) Indeed, if $s(A)<n$, there would exist an equation

$$
-1=f_{1}(x)^{2}+\cdots+f_{n-1}(x)^{2}+f_{0}(x)\left(1+x_{1}^{2}+\cdots+x_{n}^{2}\right)
$$

where $f_{j}(x) \in \mathbf{R}[x]=\mathbf{R}\left[x_{1}, \ldots, x_{n}\right]$. For $i=\sqrt{-1}$, one has $f_{j}(i x)=p_{j}(x)+$ $i q_{j}(x)$, where $p_{j}$ are $e v e n$ real polynomials and $q_{j}$ are odd real polynomials. Thus, replacing $x$ by $i x$ in (1) and comparing the real parts, one gets

$$
-1=\sum_{j=1}^{n-1}\left(p_{j}(x)^{2}-q_{j}(x)^{2}\right)+p_{0}(x)\left(1-x_{1}^{2}-\cdots-x_{n}^{2}\right) .
$$

Consider the continuous mapping $Q: \mathbf{R}^{n} \rightarrow \mathbf{R}^{n-1}$ defined by the ( $\left.n-1\right)$-tuple $\left(q_{1}, \ldots, q_{n-1}\right)$. By the Borsuk-Ulam Theorem, $Q$ must collapse some pair of antipodal points on the sphere $S^{n-1} \subset \mathbf{R}^{n}$, say $Q(-a)=Q(a), a \in S^{n-1}$. But $q_{j}(-x)=-q_{j}(x)$ for all $j$, so we also have $Q(-a)=-Q(a)$, whence $Q(a)=0$. Plugging the vector $a$ into (2), we get $-1=\sum_{j=1}^{n-1} p_{j}(a)^{2} \in \mathbf{R}$, a contradiction.

By a suitable generalization of this method, one can show that $\mathbf{R}\left[x_{1}, \ldots, x_{n}\right] /\left(1+f_{1}\left(x_{1}\right)^{2}+\cdots+f_{n}\left(x_{n}\right)^{2}\right)$ has level $n$ as long as $f_{1} f_{2} \cdots f_{n}$ $\neq 0$ and each $f_{j}(t)=0$ has a real root. Thus, for instance, in $\mathbf{R}\left[x_{1}, \ldots, x_{n}\right] /$ $\left(1+x_{1}^{2 r}+\cdots+x_{n}^{2 r}\right),-1$ is a sum of $n 2 r$ th powers, but not a sum of $n-1$ $2 r$ th powers.

We want to thank A. Kas, A. Wang and Q. M. Wang for several valuable suggestions. Details of this work will appear elsewhere. 


\section{REFERENCES}

1. R. Baeza, Quadratic forms over semilocal rings, Lecture Notes in Math., vol. 655, Springer-Verlag, Berlin and New York, 1978.

2. M. Knebusch, Some open problems, Proc. Quadratic Form Conf. (G. Orzech, ed.), Queen's Papers in Pure and Applied Math., vol. 46, Kingston, Ontario, 1976, pp. 361-370.

3. A. Pfister, Darstellung von -1 als Summe von Quadraten in einem Körper, J. London Math. Soc. 40 (1965), 159-165.

INSTITUTE OF MATHEMATICS, PEKING, CHINA

DEPARTMENT OF MATHEMATICS, UNIVERSITY OF CALIFORNIA, BERKELEY, CALIFORNIA 94720

SCHOOL OF MATHEMATICS, INSTITUTE FOR ADVANCED STUDY, PRINCETON, NEW JERSEY 08540 